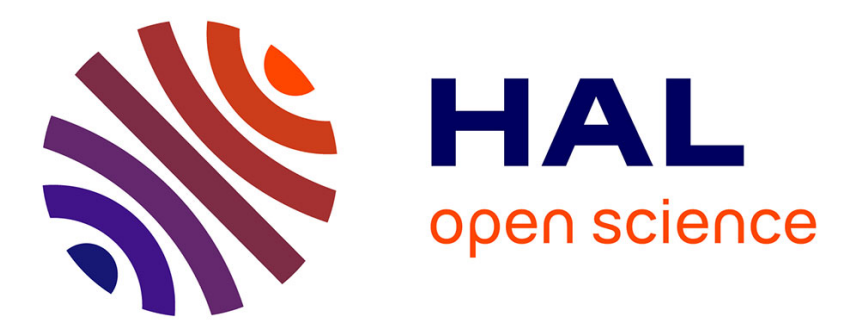

\title{
L2-stability criterion for systems with decentralized asynchronous controllers
}

Jijju Thomas, Laurentiu Hetel, Christophe Fiter, Nathan van de Wouw, Jean-Pierre Richard

\section{- To cite this version:}

Jijju Thomas, Laurentiu Hetel, Christophe Fiter, Nathan van de Wouw, Jean-Pierre Richard. L2stability criterion for systems with decentralized asynchronous controllers. 57th IEEE Conference on Decision and Control, Dec 2018, Miami Beach, Florida, United States. hal-01893547

\section{HAL Id: hal-01893547 https://hal.science/hal-01893547}

Submitted on 24 Oct 2019

HAL is a multi-disciplinary open access archive for the deposit and dissemination of scientific research documents, whether they are published or not. The documents may come from teaching and research institutions in France or abroad, or from public or private research centers.
L'archive ouverte pluridisciplinaire $\mathbf{H A L}$, est destinée au dépôt et à la diffusion de documents scientifiques de niveau recherche, publiés ou non, émanant des établissements d'enseignement et de recherche français ou étrangers, des laboratoires publics ou privés. 


\title{
$\mathcal{L}_{2}$-Stability Criterion for Systems with Decentralized Asynchronous Controllers
}

\author{
Jijju Thomas ${ }^{1}$, Laurentiu Hetel ${ }^{2}$, Christophe Fiter, ${ }^{3}$ Nathan van de Wouw ${ }^{4}$, and Jean-Pierre Richard ${ }^{5}$
}

\begin{abstract}
This paper deals with the stability analysis of decentralized sampled-data Linear Time Invariant (LTI) control systems with asynchronous sensors and actuators. We consider the case where each controller in the decentralized setting has its own sampling and actuation frequency which translates to asynchrony between sensors and actuators. The errors induced due to sampling and asynchronicity are modelled using two different operator approaches, leading to simple $\mathcal{L}_{2}$ stability criteria for the overall decentralized control system. The simplicity of the obtained criteria is illustrated by an example and simulation results exhibit the effectiveness of the approach.
\end{abstract}

\section{INTRODUCTION}

Decentralized control systems can be found aplenty in technological, environmental or societal environments $[1,2]$. In such systems, controllers are assigned to individual subsystems, using only local plant information (see Fig. 1 for a typical example). Since the feedback scheme involved in decentralized control is local, there are a few advantages of decentralization. Firstly, a substantial amount of wiring can be avoided. Secondly, owing to the decoupled nature of the controllers, the diagnosis and maintenance is easier. The aforementioned two points also translate to overall lower running costs [1], [2] and [3]. However, the design of such a decentralized control scheme may be quite complex since the local design has to be done from a global perspective. In this paper, a particular problem within the sampled-data implementation $[4,5]$ of decentralized controls is considered. More precisely, we will analyze the effect of asynchronism between the local sampled data controllers on the overall stability of the system. At an implementation level, con-

This work was supported by project UCoCoS, funded by the European Union's EU Framework Programme for Research and Innovation, Horizon H2020, Grant Agreement No: 675080. The project was also funded by ANR Project ROCC-SYS (agreement ANR-14-CE27-0008)

${ }^{1}$ Jijju Thomas(jijju.thomas@inria.fr) is with Univ. Lille, CNRS, Inria, Centrale Lille, UMR 9189 - CRIStAL, Inria Lille Nord-Europe, F-59000 Lille, France and Eindhoven University of Technology, The Netherlands

${ }^{2}$ Laurentiu Hetel (laurentiu.hetel@ec-lille.fr) is with Univ. Lille, CNRS Centrale Lille, UMR 9189 - CRIStAL - Centre de Recherche en Informatique Signal et Automatique de Lille, F-59000 Lille, France.

${ }^{3}$ Christophe Fiter (christophe.fiter@univ-lille.fr) is with Univ. Lille, CNRS, Centrale Lille, UMR 9189 - CRIStAL - Centre de Recherche en Informatique Signal et Automatique de Lille, F-59000 Lille, France.

${ }^{4}$ Nathan van de Wouw (n.v.d.wouw@tue.nl) is with Eindhoven University of Technology, Eindhoven 5600 MB, The Netherlands, with the Department of Civil, Environmental and Geo-Engineering, University of Minnesota, Minneapolis, MN 55455 USA, and also with the Delft Center for Systems and Control, Delft University of Technology, Delft 2628 CD, The Netherlands.

5 Jean-Pierre Richard (jean-pierre.richard@ec-lille.fr) is with with Univ. Lille, CNRS, Inria, Centrale Lille, UMR 9189 CRIStAL, Inria Lille NordEurope, F-59000 Lille, France.

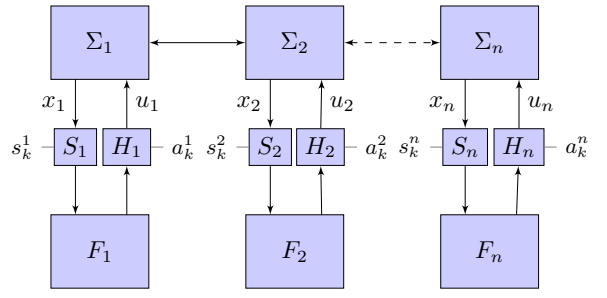

Fig. 1: A decentralized controller setup.

trollers are usually algorithms programmed on embedded processors which work at different frequencies. Moreover, sensors and actuators are distributed over different communication channels which function aperiodically. This renders the synchronization of the different elements in control loops quite challenging [6]. This may in turn affect the overall performance of the system and even its stability as illustrated in the following example. Consider the decentralized LTI system defined by

$$
\begin{array}{ll}
\Sigma_{1}: & \dot{x}_{1}(t)=-2 x_{1}(t)-x_{2}(t)+u_{1}(t) \\
\Sigma_{2}: & \dot{x}_{2}(t)=4 x_{2}(t)-2.8 x_{1}(t)+u_{2}(t)
\end{array}
$$

where $u_{1}(t)=-\hat{x}_{1}(t), u_{2}(t)=-4.6 \hat{x}_{2}(t)$ are the decentralized control inputs to systems $\Sigma_{1}$ and $\Sigma_{2}$ respectively, and $\hat{x}_{1}(t), \hat{x}_{2}(t)$ are the state values obtained through sampling and hold. In the event both systems $\Sigma_{1}$ and $\Sigma_{2}$ are sampled periodically as well as synchronously with a sampling period $T=0.59$ (i.e., $\hat{x}_{i}(t)=x_{i}(k T), \forall t \in[k T,(k+1) T), i=$ $\{1,2\})$, the overall system is stable as illustrated in Figure 2a. However, as can be seen from Figure 2b, the stability is affected when the sampling is periodic but control loops are asynchronous. Figure $2 \mathrm{~b}$ presents the case when a shift $\delta=0.2$ is introduced in the sampling of the second state, i.e., when $\hat{x}_{2}(t)=x_{2}(k T+\delta), \forall t \in[k T+\delta,(k+1) T+\delta)$. The stability problem can become even more complex when both the sensors and actuators involved within individual control loops are asynchronous.

In this paper, we will address the problem of stability analysis for the case of LTI systems with decentralized sampled-data linear controllers subject to asynchronicity. More precisely, we consider that each sampled-data controller has its own sampling and actuation frequencies. This particular problem setting gives rise to complexities induced by sampling, asynchronicity, network effects, etc. The decentralized control problem that we introduce in this paper is unique to the best of our knowledge.

Although the problem considered in this paper is novel, stability analysis methods have been proposed for centralized 


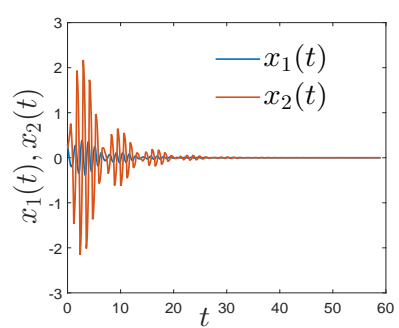

(a)

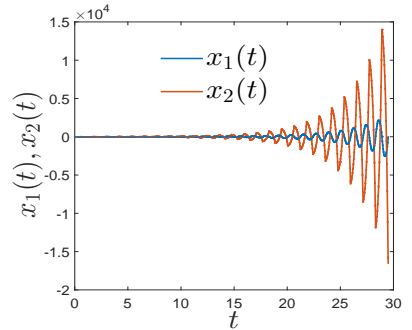

(b)
Fig. 2: (a) The decentralized LTI system (1) is stable for synchronous sampling with $T=0.59$. (b) The stability is affected when $x_{2}(t)$ is sampled asynchronously with respect to $x_{1}(t)$ with a shift of $\delta=0.2$.

controllers subjected to sampling and asynchronism between sensors and actuators [7, 8, 9]. However, the period between sampling and actuation instants was treated to be constant and all the system state data was considered to be sampled at same time instants. In the scope of sampled-data and networked control systems [10, 11, 12], there are a few similarities with the problem we consider. For example, decentralized event-triggered control and delay introduced due to network effects can also be seen as a form of asynchronism [13]. In comparison to the very few existing results addressing problems similar to the one considered in this paper, we propose a novel and simple approach that guarantees stability.

The main contribution of this paper is to provide approaches for $\mathcal{L}_{2}$-stability analysis of decentralized sampleddata controllers. For the sake of generality, we consider the sampling and actuation intervals to be time-varying and possibly unknown (but bounded). We take into account the asynchronicity between individual controllers as well as the asynchronicity between sensors and actuators within a local control loop. By using tools based on input-output methods $[14,15]$, related to the ones previously used for systems with delays [16, 17, 18, 19], we provide two novel and different stability analysis methods based on easy-to-check frequencydomain criteria.

The remainder of this paper has been structured as follows. In Section II, we introduce the problem formulation, followed by technical preliminaries. Sections III and IV deal with the transformation of the closed-loop sampled-data dynamics into a feedback interconnection model and provide a stability criterion. Section $\mathrm{V}$ provides a numerical example corroborating the presented results.

Notations: $\mathbb{R}$ is the set of all real numbers, implying $\mathbb{R}^{n}$ is the set of all $n$-dimensional real vectors. $\operatorname{Diag}\left(M_{1}, M_{2}, \ldots, M_{n}\right)$ is the block diagonal matrix with elements $M_{i}$ of appropriate dimensions. $\mathcal{L}_{2 e}(a, b)$ is the extended $\mathcal{L}_{2}$-space of all square integrable and Lebesgue measurable functions defined on the interval $[a, b]$, with the $\mathcal{L}_{2}$-norm defined as $\|q\|_{\mathcal{L}_{2}}^{2}=\langle q, q\rangle$, and the inner product $\langle p, q\rangle=\int_{a}^{b} p(s)^{T} q(s) d s$.

\section{PROBLEM FORMULATION AND TECHNICAL PRELIMINARIES}

\section{A. Motivating Problem}

1) System Model: The system under consideration consists of a set of Linear Time Invariant (LTI) systems, wherein each individual system is influenced by its corresponding control input and other system states. Figure 1 depicts this decentralized control configuration. Consider that the dynamics of each LTI system (denoted $\Sigma_{i}$ ) is given by

$$
\dot{x}_{i}(t)=A_{i} x_{i}(t)+B_{i} u_{i}(t)+\sum_{j=1, i \neq j}^{M} A_{i j} x_{j}(t), \forall t \in \mathbb{R},
$$

with $i \in\{1,2, . ., M\}, x_{i}(t) \in \mathbb{R}^{n_{i}}$ and $u_{i}(t) \in \mathbb{R}^{m_{i}}$. The matrices $A_{i}, B_{i}$ and $A_{i j}$ are of appropriate dimensions. The term $A_{i j} x_{j}(t)$ denotes the influence of the states of the $j^{\text {th }}$ plant $\Sigma_{j}$ on the dynamics of system $\Sigma_{i}$. Here, we consider the case where the control of the global system is linear. Furthermore, we assume that it is decentralized in the sense that the control input $u_{i}(t)$ only depends on the local state variables $x_{i}(t)$. Furthermore, we consider that the control inputs are asynchronous. The system states $x_{i}(t)$ are sampled according to a sampling sequence $\left\{s_{k}^{i}\right\}_{k \in \mathbb{Z}}$ defined by

$$
\left\{s_{k}^{i}: s_{k+1}^{i}-s_{k}^{i}=h_{k}^{i}, k \in \mathbb{Z}, i \in\{1,2, . ., M\}\right\} .
$$

The sequence of sampling intervals $\left\{h_{k}^{i}\right\}_{k \in \mathbb{Z}}$ satisfying $h_{k}^{i} \in\left[\underline{h}_{i}, \bar{h}_{i}\right]$ takes into account imperfection in sampling caused by e.g. jitter, data packet dropouts, etc. Note that the sampling instants of different systems are not necessarily synchronous. The control input $u_{i}(t)$ based on $x_{i}\left(s_{k}^{i}\right)$ will be implemented at a time instant $a_{k}^{i}$ at the level of the actuator of system $\Sigma_{i}$. We consider that the sequence of actuation times $\left\{a_{k}^{i}\right\}_{k \in \mathbb{Z}}$ satisfies

$$
\left\{a_{k}^{i}: a_{k}^{i}=s_{k}^{i}+\eta_{k}^{i}, \eta_{k}^{i} \leq h_{k}^{i}, k \in \mathbb{Z}, i \in\{1,2, \ldots, M\}\right\},
$$

where $\eta_{i}^{k} \in\left[\eta_{i}, \bar{\eta}_{i}\right]$ denotes the asynchrony between sensors and actuators. Such an asynchrony may be due to network delays, control computation delay, etc. Based on this consideration, the control input to the system $\Sigma_{i}$ is given by the sampled-data decentralized static state-feedback law

$$
u_{i}(t)=F_{i} x_{i}\left(s_{k}^{i}\right), \quad \forall t \in\left[a_{k}^{i}, a_{k+1}^{i}\right) .
$$

The goal of this paper is to analyse the stability of the system defined by (2), (3), (4) and (5) ${ }^{1}$.

\section{B. Preliminaries}

We introduce some basic concepts of linear operator theory that are used in this paper. An operator $\mathbf{G}: \mathcal{L}_{2 e}(a, b) \rightarrow$ $\mathcal{L}_{2 e}(c, d)$ receives an input $p \in \mathcal{L}_{2 e}(a, b)$ and produces an output $q \in \mathcal{L}_{2 e}(c, d)$.

\footnotetext{
${ }^{1}$ The exact mathematical concept of stability that we use in this paper will be formalized in Section II-B
} 


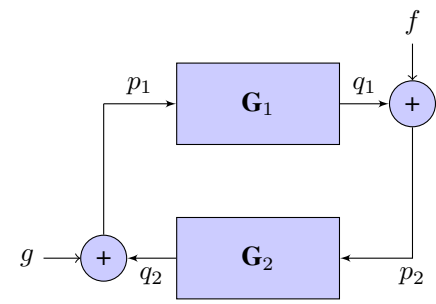

Fig. 3: Standard feedback interconnection of operators $\mathbf{G}_{1}$ and $\mathbf{G}_{2}$.

1) Bounded operators: The operator $\mathbf{G}: \mathcal{L}_{2 e}(a, b) \rightarrow$ $\mathcal{L}_{2 e}(c, d)$ is said to be bounded if there exists a constant $\gamma \in \mathbb{R}$ so that $\|\mathbf{G}(p)\|_{\mathcal{L}_{2}} \leq \gamma\|p\|_{\mathcal{L}_{2}}$ for all $p \in \mathcal{L}_{2 e}(a, b)$. The minimal constant $\gamma$ satisfying the aforementioned inequality is called the induced $\mathcal{L}_{2}$-gain of the operator $\mathbf{G}$, and is denoted by $\|\mathbf{G}\|_{\mathcal{L}_{2}}$ or $\gamma(G)$.

2) Feedback interconnection: The standard feedback interconnection of two operators $\mathbf{G}_{1}$ and $\mathbf{G}_{2}$, is given by

$$
\Sigma_{\mathbf{G}_{1} \mathbf{G}_{2}}:\left\{\begin{array}{l}
p_{2}=\mathbf{G}_{1} p_{1}+f \\
p_{1}=\mathbf{G}_{2} p_{2}+g .
\end{array}\right.
$$

Figure 3 shows the graphical representation of the standard feedback interconnection.

3) Well-posed system: A feedback system is said to be well-posed if all the closed-loop transfer matrices are welldefined and proper [20]. The implication for the standard feedback interconnection $\Sigma_{\mathbf{G}_{1} \mathbf{G}_{2}}$ given by (6) is that the well-posedness is guaranteed only if $I-\mathbf{G}_{1} \mathbf{G}_{2}$ is invertible.

4) $\mathcal{L}_{2}$-stability: An operator $\mathbf{G}$ is said to be $\mathcal{L}_{2}$-stable if it has a finite $\mathcal{L}_{2}$-gain [21].

5) Small-Gain Theorem: A feedback interconnection of two operators $\mathbf{G}_{\mathbf{1}}$ and $\mathbf{G}_{\mathbf{2}}$ given by (6), has a finite $\mathcal{L}_{2}$-gain for the mapping

$$
\left[\begin{array}{l}
f \\
g
\end{array}\right] \rightarrow\left[\begin{array}{l}
p_{1} \\
p_{2}
\end{array}\right]
$$

if

$$
\gamma\left(\mathbf{G}_{\mathbf{1}}\right) \gamma\left(\mathbf{G}_{\mathbf{2}}\right)<1,
$$

where $\gamma\left(\mathbf{G}_{\mathbf{1}}\right)$ and $\gamma\left(\mathbf{G}_{\mathbf{2}}\right)$ are the $\mathcal{L}_{2}$-gain of the operators $\mathbf{G}_{\mathbf{1}}$ and $\mathbf{G}_{\mathbf{2}}$ respectively [22].

In this paper, we will use an operator approach to take into account the asynchrony in decentralized control loops. Stability will be analysed in the $\mathcal{L}_{2}$-sense by modelling the system and the effects of sampling and asynchrony using operators. Two methods will be presented. The first method models the overall effect of sampling and asynchrony between sensors and actuators in a global manner using one operator. The second method takes the difference between the effects of sampling and asynchrony using two separate operators.

\section{STABILITY ANALYSIS USING A SINGLE OPERATOR FOR SAMPLING AND ASYNCHRONY}

The configuration shown in Figure 1, defined by (2) can also be expressed by the standard state-space equation

$$
\dot{X}(t)=A X(t)+B U(t), \forall t \in \mathbb{R},
$$

wherein the system state $X(t) \in \mathbb{R}^{n}$ and the control input $U(t) \in \mathbb{R}^{m}$ can be decomposed as

$$
\begin{aligned}
X(t) & =\left[\begin{array}{llll}
x_{1}^{T}(t) & x_{2}^{T}(t) & \ldots & x_{M}^{T}(t)
\end{array}\right]^{T}, \\
U(t) & =\left[\begin{array}{llll}
u_{1}^{T}(t) & u_{2}^{T}(t) & \ldots & u_{M}^{T}(t)
\end{array}\right]^{T} .
\end{aligned}
$$

with $x_{i}(t) \in \mathbb{R}^{n_{i}}, u_{i}(t) \in \mathbb{R}^{m_{i}}, \sum_{i=1}^{M} n_{i}=n$ and $\sum_{i=1}^{M} m_{i}=m$. Similarly, the system matrix $A \in \mathbb{R}^{n \times n}$ and the input matrix $B \in \mathbb{R}^{n \times m}$ are given by

$$
\begin{aligned}
A & =\left[\begin{array}{cccc}
A_{1} & A_{12} & \ldots & A_{1 M} \\
A_{21} & A_{2} & \ldots & A_{2 M} \\
\vdots & \vdots & \ddots & \vdots \\
A_{M 1} & A_{M 2} & \ldots & A_{M}
\end{array}\right], \\
B & =\operatorname{diag}\left(B_{1}, B_{2}, \ldots, B_{M}\right) .
\end{aligned}
$$

\section{A. System Model Reformulation}

Let $\hat{x}_{i}(t)$ represent the information used in computing the control input $u_{i}(t)$ under the influence of asynchrony and sampling:

$$
\begin{aligned}
\hat{x}_{i}(t) & =x_{i}\left(s_{k}^{i}\right), \forall t \in\left[a_{k}^{i}, a_{k+1}^{i}\right), \\
\hat{X}(t) & =\left[\begin{array}{llll}
\hat{x}_{1}^{T}(t) & \hat{x}_{2}^{T}(t) & \ldots & \hat{x}_{M}^{T}(t)
\end{array}\right]^{T}
\end{aligned}
$$

with $k \in \mathbb{Z}$ and $i \in\{1,2, \ldots, M\}$. Consequently, the decentralized control law can be defined as

$$
U(t)=F \hat{X}(t)
$$

with $F=\operatorname{diag}\left(F_{1}, F_{2}, \ldots, F_{M}\right)$. The closed-loop system model defined by (2), (3), (4), (5) can, therefore, be formulated as follows:

$$
\begin{aligned}
\dot{X}(t) & =A X(t)+B F \hat{X}(t) \\
& =(A+B F) X(t)+B F(\hat{X}(t)-X(t)) \\
& =A_{c l} X(t)+B_{c l} E(t),
\end{aligned}
$$

where $A_{c l}:=A+B F$ and $B_{c l}:=B F$. The vector $E(t)$ in (14) represents the error induced in the system (9) by sampling and asynchrony, i.e,

$$
E(t):=\hat{X}(t)-X(t)=\left[\begin{array}{llll}
e_{1}^{T}(t) & e_{2}^{T}(t) & \ldots & e_{M}^{T}(t)
\end{array}\right]^{T},
$$

where

$$
\begin{aligned}
e_{i}(t) & =\hat{x}_{i}(t)-x_{i}(t), \\
& =x_{i}\left(s_{k}^{i}\right)-x_{i}(t) .
\end{aligned}
$$

Choosing an auxiliary output

$$
\begin{aligned}
Y(t)=\dot{X}(t) & =\left[\begin{array}{llll}
\dot{x}_{1}(t)^{T} & \dot{x}_{2}(t)^{T} & \ldots & \dot{x}_{M}(t)^{T}
\end{array}\right]^{T}, \\
& =\left[\begin{array}{llll}
y_{1}^{T}(t) & y_{2}^{T}(t) & \ldots & y_{M}^{T}(t)
\end{array}\right]^{T}
\end{aligned}
$$

and by using an integral operator $\Delta_{i}: \mathcal{L}_{2 e}^{n_{i}}(-\infty, \infty) \rightarrow$ $\mathcal{L}_{2 e}^{n_{i}}(-\infty, \infty)$, we can rewrite (16) as follows:

$e_{i}(t)=\left(\Delta_{i} y_{i}\right)(t):=-\int_{s_{k}^{i}}^{t} y_{i}(\theta) d \theta, \forall t \in\left[a_{k}^{i}, a_{k+1}^{i}\right), k \in \mathbb{Z}$.

The operator $\Delta_{i}$ accounts for the error induced in the system $\Sigma_{i}$ (in closed-loop with its local controller) by sampling and asynchrony. 


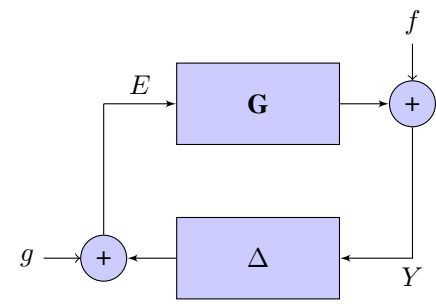

Fig. 4: The system (14) as a feedback interconnection

\section{B. Stability Analysis}

Motivated by the problem under consideration in Section II-A, we study the stability of the feedback interconnection of $\mathbf{G}$ and $\Delta$ in the standard form (see Figure 4), defined by

$$
\Sigma_{\mathbf{G} \Delta}:\left\{\begin{array}{l}
Y=\mathbf{G} E+f \\
E=\Delta Y+g,
\end{array}\right.
$$

where $f, g \in \mathcal{L}_{2 e}^{n}(-\infty, \infty)$. The operator $\mathbf{G}$ is defined by the transfer function

$$
G(s)=C_{c l}\left(s I-A_{c l}\right)^{-1} B_{c l}+D_{c l},
$$

where

$$
\begin{aligned}
& C_{c l}=A_{c l}=A+B F, \\
& D_{c l}=B_{c l}=B F,
\end{aligned}
$$

and the operator $\Delta=\operatorname{diag}\left(\Delta_{1}, \Delta_{2}, \ldots, \Delta_{M}\right)$, where $\Delta_{i}$ is defined by (18). The feedback interconnection $\Sigma_{\mathbf{G} \Delta}$ is equivalent to the decentralized control system given by (2), (3), (4) and (5), affected by perturbations in the measured state value. That is,

$$
\dot{x}_{i}(t)=A_{i} x_{i}(t)+B_{i} F_{i}\left(x_{i}\left(s_{k}^{i}\right)+w_{i}(t)\right)+\sum_{j=1, i \neq j}^{M} A_{i j} x_{j}(t),
$$$$
w_{i}(t)=g_{i}(t)+\int_{s_{k}^{i}}^{t} f_{i}(s) d s, x_{i}(0)=0 .
$$

$f_{i}$ and $g_{i}$ are components of $f$ and $g$, with appropriate dimensions. Before providing the stability criterion, we introduce the following technical lemma which is an adaptation of the result in [17] for continuous-time systems with time delay.

Lemma 1: The $\mathcal{L}_{2}$ induced norm of the operator $\Delta$ is upper-bounded by $\gamma$, where

$$
\gamma=\max _{i=1}^{M}\left\{\bar{h}_{i}+\bar{\eta}_{i}\right\}
$$

[23].

Proof: The proof is available in the technical report

Using Lemma 1, the following stability result can be obtained.

Theorem 2: The feedback interconnection of operators $\mathbf{G}$ and $\Delta$, denoted by $\Sigma_{\boldsymbol{G} \Delta}$ in (19) is $\mathcal{L}_{2}$-stable if

$$
\sup _{\omega \in \mathbb{R}} \bar{\sigma}(G(j \omega))<\left(\max _{i=1}^{M}\left\{\bar{h}_{i}+\bar{\eta}_{i}\right\}\right)^{-1},
$$

where $G(j \omega)=C_{c l}\left(j \omega I-A_{c l}\right)^{-1} B_{c l}+D_{c l}$ is the frequency response function matrix of the system defined by (14) and (17), with the matrices $A_{c l}, B_{c l}, C_{c l}$ and $D_{c l}$ defined in (21), and $\bar{\sigma}(G(j \omega))$ is the largest singular value of the $G(j \omega)$ [23].

Proof: The proof is available in the technical report

\section{STABILITY ANALYSIS USING TWO SEPARATE OPERATORS}

We have seen that Theorem 2 provides an easy-to-check criterion for stability analysis of the closed-loop LTI system (9), since it only requires a frequency-domain check of an LTI system. However, this result may be conservative since both the effects of sampling and asynchrony are modelled using a global operator. Below, we propose an alternative approach in which the error induced by the sampling and asynchrony are considered separately, in terms of an operator that represents the effect of sampling and hold, and an operator that represents the delay induced by asynchrony. This alleviates conservatism by providing flexibility in employing more accurate function bounding inequalities.

\section{A. Feedback interconnection system representation}

In this section, we show that the operator $\Delta$ can be decomposed into two separate operators. The operator $\Delta^{\text {sam }}$ represents the error induced by sampling whereas the operator $\Delta^{a s y}$ denotes the error induced by asynchrony between the sensors and actuators. Let us recall the definition of $E(t)$ defined as in (15). Let $\tilde{x}_{i}(t)$ denote the sampled version of $x_{i}(t)$, along the sampling sequence $\left\{s_{k}^{i}\right\}_{k \in \mathbb{Z}}$ and be given by

$$
\tilde{x}_{i}(t)=x_{i}\left(s_{k}^{i}\right) \quad \forall t \in\left[s_{k}^{i}, s_{k+1}^{i}\right) .
$$

We care to stress the difference between $\tilde{x}_{i}(t)$ in (25) and $\hat{x}_{i}(t)$ in (12) in terms of their domain of definition. Let us define

$$
e_{i}^{s a m}(t):=\tilde{x}_{i}(t)-x_{i}(t), \quad \forall t \in\left[s_{k}^{i}, s_{k+1}^{i}\right) .
$$

Note that $e_{i}^{s a m}(t)$ corresponds to the error between the signal $x(t)$ and it's sampled version (see Figure 5 for a graphical illustration). Given the signal $y_{i}(t)$ in (17), the sampling induced error can be characterized by

$$
e_{i}^{s a m}(t)=-\int_{s_{k}^{i}}^{t} y_{i}(\theta) d \theta=:\left(\Delta_{i}^{s a m} y_{i}\right)(t) .
$$

Considering the rectangular signal $\tilde{x}_{i}(t)$, representing the sampled version of system state $x_{i}(t)$, and the signal $\hat{x}_{i}(t)$ as given in (12) representing the signal actually used at the level of actuators, the effect of asynchrony can be captured by introducing an error

$$
e_{i}^{a s y}(t)=\hat{x}_{i}(t)-\tilde{x}_{i}(t), \quad \forall t \geq 0
$$

as illustrated in Figure 6. Let us remark that

$$
e_{i}^{a s y}(t):= \begin{cases}x_{i}\left(s_{k-1}^{i}\right)-x_{i}\left(s_{k}^{i}\right), & \forall t \in\left[s_{k}^{i}, a_{k}^{i}\right) \\ 0, & \forall t \in\left[a_{k}^{i}, s_{k+1}^{i}\right)\end{cases}
$$

Considering $y_{i}(t)$ as given in (17), we define

$$
\begin{aligned}
e_{i}^{a s y}(t) & =\left(\Delta_{i}^{a s y} y\right)(t) \\
& := \begin{cases}-\int_{s_{k-1}^{i}}^{s_{k}^{i}} y_{i}(\theta) d \theta, & \forall t \in\left[s_{k}^{i}, a_{k}^{i}\right) \\
0, & \forall t \in\left[a_{k}^{i}, s_{k+1}^{i}\right) .\end{cases}
\end{aligned}
$$



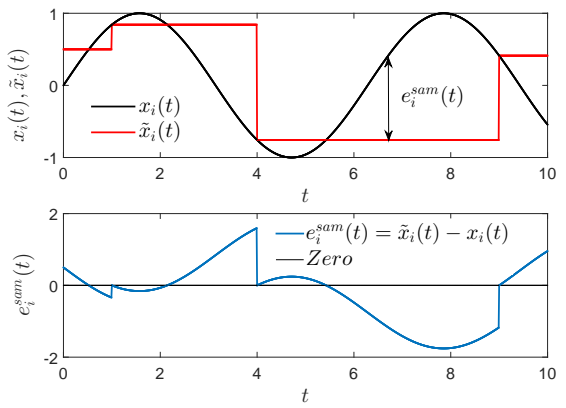

Fig. 5: The top figure gives a sample signal $x_{i}(t)$ (in black) and its sampled version $\tilde{x}_{i}(t)$ (in red). The bottom figure shows the resetting nature (to zero) of the sampling-induced error $e_{i}^{s a m}(t)$ for the given $x_{i}(t)$ and $\tilde{x}_{i}(t)$.
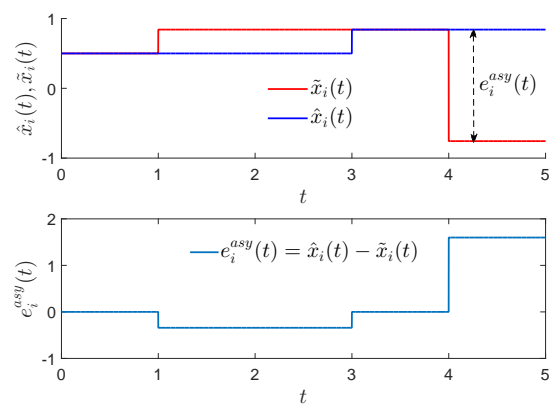

Fig. 6: The top figure gives the sampled signal $\tilde{x}_{i}(t)$ (in red) and the version $\hat{x}_{i}(t)$ (in blue) that is used at the actuator level. The bottom figure shows the piece-wise constant error $e_{i}^{a s y(t)}$ introduced due to asynchrony for the given $x_{i}(t)$, $\tilde{x}_{i}(t)$ and $\hat{x}_{i}(t)$.

Since

$$
\begin{aligned}
& \tilde{x}_{i}(t)=x_{i}(t)+e_{i}^{s a m}(t), \\
& \hat{x}_{i}(t)=\tilde{x}_{i}(t)+e_{i}^{a s y}(t),
\end{aligned}
$$

we have $\hat{x}_{i}(t)=x_{i}(t)+e_{i}^{s a m}(t)+e_{i}^{a s y}(t)$, which leads to the decomposition of $\Delta_{i}$ in (18) given by

$$
\left(\Delta_{i} y_{i}\right)(t)=\left(\Delta_{i}^{s a m} y_{i}\right)(t)+\left(\Delta_{i}^{a s y} y_{i}\right)(t)
$$

as shown in Figure 7. Then, we have for the reformulated system (14) and (17),

$$
E(t)=\left[\begin{array}{llll}
e_{1}^{T}(t) & e_{2}^{T}(t) & \ldots & e_{M}^{T}(t)
\end{array}\right]^{T}
$$

with

$$
\begin{aligned}
e_{i}(t) & =\left(\Delta_{i} y\right)(t) \\
& =\left(\Delta_{i}^{s a m} y\right)(t)+\left(\Delta_{i}^{a s y} y\right)(t), \quad \forall t \in\left[s_{k}^{i}, s_{k+1}^{i}\right) .
\end{aligned}
$$

\section{B. Stability Analysis}

In the following lemma, we compute the $\mathcal{L}_{2}$-norm of the operator $\Delta_{i}$ by upper-bounding each of the operators introduced by the decomposition shown in (34), thereby providing a bound on the operator $\Delta$.

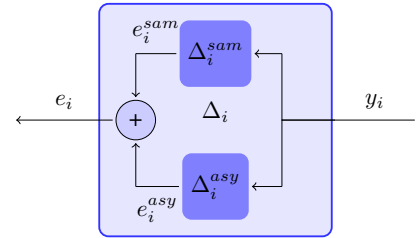

Fig. 7: The decomposition of operator $\Delta_{i}$ into operators $\Delta_{i}^{s a m}$ and $\Delta_{i}^{a s y}$, that introduce the sampling error $e_{i}^{s a m}$ and asynchrony error $e_{i}^{a s y}$ respectively.

Lemma 3: The $\mathcal{L}_{2}$-induced norm of the operator $\Delta$ is upper-bounded by $\gamma_{1}$, where

$$
\gamma_{1}=\max _{i=1}^{M}\left\{\frac{2 \bar{h}_{i}}{\pi}+\sqrt{\bar{h}_{i} \bar{\eta}_{i}}\right\} .
$$
[23].

Proof: The proof is available in the technical report

Based on Lemma 3, we provide in Theorem 4 a less conservative and also easy-to-check stability criterion for the $\mathcal{L}_{2}$-stability of the feedback interconnection $\Sigma_{\mathbf{G} \Delta}$.

Theorem 4: The feedback interconnection $\Sigma_{\boldsymbol{G} \Delta}$ of operators $\mathbf{G}$ and $\Delta$ as defined in (19), where $\Delta$ satisfies the decomposition (34), is $\mathcal{L}_{2}$-stable if

$$
\sup _{\omega \in \mathbb{R}} \bar{\sigma}(G(j \omega))<\left(\max _{i=1}^{M}\left\{\frac{2 \bar{h}_{i}}{\pi}+\sqrt{\bar{h}_{i} \bar{\eta}_{i}}\right\}\right)^{-1},
$$

where $G(j \omega)=C_{c l}\left(j \omega I-A_{c l}\right)^{-1} B_{c l}+D_{c l}$ is the frequency response function matrix of the closed-loop system defined by (14) and (17), with the matrices $A_{c l}, B_{c l}, C_{c l}$ and $D_{c l}$ defined in (21).

Proof: The proof is available in the technical report [23].

\section{SIMULATION RESULTS}

In this section, we apply the stability criteria provided in Theorems 2 and 4 to the decentralized LTI system previously considered in Section I, equation (1). Expressing the decentralized system (1) in the standard state-space model given by (9), we have

$$
A=\left[\begin{array}{cc}
-2 & -1 \\
2.8 & 4
\end{array}\right], B=\left[\begin{array}{ll}
1 & 0 \\
0 & 1
\end{array}\right], F=\left[\begin{array}{cc}
-1 & 0 \\
0 & -4.6
\end{array}\right],
$$

which provides

$$
\begin{aligned}
& A_{c l}=C_{c l}=A+B F=\left[\begin{array}{cc}
-3 & -1 \\
2.8 & -0.6
\end{array}\right], \\
& B_{c l}=D_{c l}=B F=\left[\begin{array}{cc}
-1 & 0 \\
0 & -4.6
\end{array}\right] .
\end{aligned}
$$

The $\mathcal{L}_{2}$-norm of the operator $\mathbf{G}$ can easily be obtained from the transfer function in (20) and is 5.2143. By employing Theorem 2, we can state that the system remains stable for all $\bar{h}_{i}$ and $\bar{\eta}_{i}$ satisfying

$$
\max _{i=1}^{2}\left\{\bar{h}_{i}+\bar{\eta}_{i}\right\}<\frac{1}{5.2143} .
$$

Similarly, using Theorem $4 \mathcal{L}_{2}$-stability is guaranteed if

$$
\max _{i=1}^{2}\left\{\frac{2 \bar{h}_{i}}{\pi}+\sqrt{\bar{h}_{i} \bar{\eta}_{i}}\right\}<\frac{1}{5.2143} .
$$




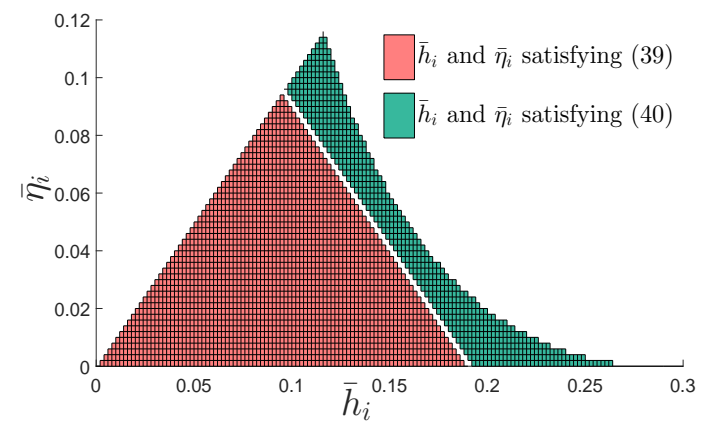

Fig. 8: $\bar{h}_{i}$ and $\bar{\eta}_{i}$ satisfying the stability criteria obtained using Theorem 2 (39) and Theorem 4 (40).

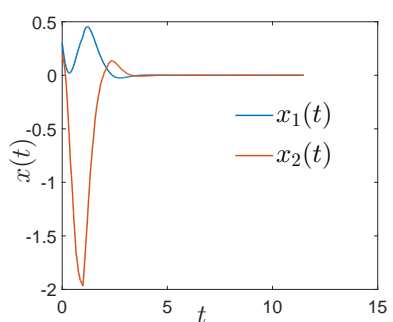

(a)

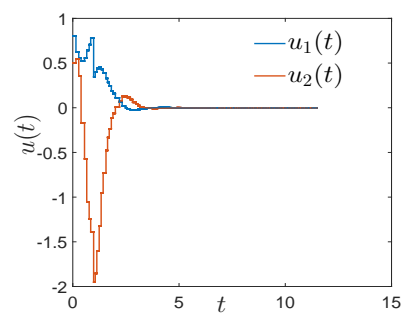

(b)
Fig. 9: The evolution of (a) system states and (b) control input for (37), with parameters $\bar{h}_{1}, \bar{h}_{2}, \bar{\eta}_{1}$ and $\bar{\eta}_{2}$ chosen according to the feasibility region shown in Figure 8.

The feasible values of $\bar{h}_{i}$ and $\bar{\eta}_{i}$ satisfying (39) and (40) are shown in Figure 8. It is quite clear that the criterion obtained using Theorem 4, given by (40), provides less conservative results in comparison to the criterion obtained using Theorem 2, given by (39). This corroborates our theoretical result that by encompassing the error induced due to sampling and asynchrony within two separate operators, we obtain a less conservative result. Additionally, the criterion is simple to employ since the only computation involved is in obtaining the $\mathcal{L}_{2}$-norm of the system operator, which can be computed easily using the $H_{\infty}$ norm of it's transfer function [24]. Choosing the parameters $\bar{h}_{1}=0.117$, $\bar{h}_{2}=0.1035, \bar{\eta}_{1}=0.0945$ and $\bar{\eta}_{2}=0.0405$, we simulate the system by introducing a rectangular wave perturbation $w_{i}(t)=0.5, \forall t \leq a_{10}^{i}, i \in\{1,2\}$. We can see in Figure 9a that the system (37) is indeed stable.

\section{CONCLUSION}

In this paper, the stability analysis problem for LTI systems with decentralized sampled-data linear controllers subjected to asynchrony has been studied. Two different stability analysis methods based on easy-to-check frequency-domain criteria have been provided. The method primarily included modelling the error induced by sampling and asynchrony using operators, and obtaining the $\mathcal{L}_{2}$-gain bounds on these operators. The effectiveness of the method was illustrated using numerical simulations.

\section{REFERENCES}

[1] L. Bakule and J. Lunze, Decentralized Design of Feedback Control for Large-scale Systems, ser. Kybernetika: Př́loha. Academia, 1988.
[2] L. Bakule, "Decentralized control: An overview," Annual Reviews in Control, vol. 32, no. 1, pp. $87-98,2008$.

[3] J. Lunze, "Feedback control of large-scale systems," International Journal of Adaptive Control and Signal Processing, vol. 11, no. 2, pp. 167-168, 1997.

[4] T. Chen and B. A. Francis, Optimal Sampled-Data Control Systems. Secaucus, NJ, USA: Springer-Verlag New York, Inc., 1995.

[5] K. Åström and B. Wittenmark, Computer-Controlled Systems: Theory and Design, Third Edition, ser. Dover Books on Electrical Engineering. Dover Publications, 2011.

[6] N. M. Freris, S. R. Graham, and P. R. Kumar, "Fundamental limits on synchronizing clocks over networks," IEEE Transactions on Automatic Control, vol. 56, no. 6, pp. 1352-1364, June 2011.

[7] M. Wakaiki, K. Okano, and J. P. Hespanha, "Stabilization of systems with asynchronous sensors and controllers," Automatica, vol. 81, pp. $314-321,2017$.

[8] M. Wakaiki, K. Okano, and J. Hespanha, "Stabilization of networked control systems with clock offsets," Proceedings of the American Control Conference, vol. 2015, pp. 3522-3527, 072015.

[9] M. Wakaiki, K. Okano, and J. P. Hespanha, "Control under clock offsets and actuator saturation," 2015 54th IEEE Conference on Decision and Control (CDC), pp. 6886-6891, Dec 2015.

[10] W. P. M. H. Heemels, A. R. Teel, N. van de Wouw, and D. Nesic, "Networked control systems with communication constraints: Tradeoffs between transmission intervals, delays and performance," IEEE Transactions on Automatic Control, vol. 55, no. 8, pp. 1781-1796, Aug 2010.

[11] L. Hetel, C. Fiter, H. Omran, A. Seuret, E. Fridman, J. P. Richard, and S. I. Niculescu, "Recent developments on the stability of systems with aperiodic sampling: An overview," Automatica, vol. 76, pp. 309 $-335,2017$.

[12] C. Fiter, T. E. Korabi, L. Etienne, and L. Hetel, "Stability of LTI systems with distributed sensors and aperiodic sampling," in Lecture Notes in Control and Information Sciences, Control subject to Computational and Communication Constraints: Current Challenges, Springer, To appear.

[13] V. S. Dolk, D. P. Borgers, and W. P. M. H. Heemels, "Output-based and decentralized dynamic event-triggered control with guaranteed $\mathcal{L}_{p}$ gain performance and zeno-freeness," IEEE Transactions on Automatic Control, vol. 62, no. 1, pp. 34-49, Jan 2017.

[14] A. Megretski and A. Rantzer, "System analysis via integral quadratic constraints," IEEE Transactions on Automatic Control, vol. 42, no. 6 , pp. 819-830, Jun 1997.

[15] G. Zames, "On the input-output stability of time-varying nonlinear feedback systems part one: Conditions derived using concepts of loop gain, conicity, and positivity," IEEE Transactions on Automatic Control, vol. 11, no. 2, pp. 228-238, Apr 1966.

[16] M. Jun and M. G. Safonov, "Iqc robustness analysis for time-delay systems," International Journal of Robust and Nonlinear Control, vol. 11, no. 15, pp. 1455-1468, 2001.

[17] C.-Y. Kao and B. Lincoln, "Simple stability criteria for systems with time-varying delays," Automatica, vol. 40, no. 8, pp. 1429 - 1434 , 2004.

[18] C.-Y. Kao and A. Rantzer, "Stability analysis of systems with uncertain time-varying delays," Automatica, vol. 43, no. 6, pp. 959 - 970, 2007.

[19] E. Fridman and U. Shaked, "Input-output approach to stability and 12gain analysis of systems with time-varying delays," Systems \& Control Letters, vol. 55, no. 12, pp. 1041 - 1053, 2006.

[20] K. Zhou and J. C. Doyle, Essentials of Robust Control. Prentice-Hall, 1998.

[21] A. J. V. d. Schaft, L2-Gain and Passivity Techniques in Nonlinear Control, 1st ed. Secaucus, NJ, USA: Springer-Verlag New York, Inc., 1996.

[22] H. K. Khalil, Nonlinear systems; 3rd ed. Prentice-Hall, 2002.

[23] J. Thomas, L. Hetel, C. Fiter, N. van de Wouw, and J. P. Richard, " $\mathcal{L}_{2}$-stability criterion for systems with decentralized asynchronous controllers," Tech. Rep., September 2018, available at https://drive. google.com/open?id=1QwLOEWwU_FARf1tP4VUydRsrpjcXrxdb.

[24] K. Zhou, J. C. Doyle, and K. Glover, Robust and Optimal Control. Upper Saddle River, NJ, USA: Prentice-Hall, Inc., 1996. 